Annuaire suisse de politique de développement

24-1 | 2005

Faits et statistiques 2005

\title{
11. Politique de paix et politique de sécurité
}

\author{
Xavier Tschumi Canosa
}

\section{OpenEdition}

\section{Journals}

Édition électronique

URL : http://journals.openedition.org/aspd/433

DOI : $10.4000 /$ aspd.433

ISSN : 1663-9669

\section{Éditeur}

Institut de hautes études internationales et du développement

\section{Édition imprimée}

Date de publication : 1 avril 2005

Pagination : 175-185

ISSN : 1660-5934

\section{Référence électronique}

Xavier Tschumi Canosa, «11. Politique de paix et politique de sécurité », Annuaire suisse de politique de développement [En ligne], 24-1 | 2005, mis en ligne le 02 mars 2010, consulté le 08 septembre 2020. URL : http://journals.openedition.org/aspd/433 ; DOI : https://doi.org/10.4000/aspd.433 


\section{Politique de paix et politique de sécurité*}

D EPUIS le début des années 2000, la politique extérieure de la Suisse donne une importance particulière à la notion de sécurité humaine et, dans cette ligne, la Confédération a mis au point la stratégie 2004-2007 de son engagement en matière de mines antipersonnel. A ce propos, la $1^{r e}$ Conférence d'examen de la convention d'Ottawa sur l'interdiction de ces mines s'est déroulée à Nairobi en novembre et décembre 2004.

Concernant la maîtrise des armements et le désarmement, la Suisse a défini sa politique en la matière dans un rapport en septembre 2004 et a continué de s'impliquer dans le désarmement chimique, notamment en Russie. La Suisse tient aussi un rôle actif au sein du Partenariat pour la paix, surtout dans le domaine des technologies de l'information.

Dans sa politique de paix, la Suisse s'est engagée au sein de la nouvelle force militaire européenne en Bosnie-Herzégovine (EUFOR), qui a remplacé la force de stabilisation de l'OTAN (SFOR) depuis décembre 2004. Avalisée par une résolution du Conseil de sécurité de l'ONU, cette force a une mission militaire et civile.

\subsection{Politique de paix}

\subsubsection{Engagement de militaires suisses en Bosnie-Herzégovine}

Fin mai 2004, le Conseil fédéral a remis au Parlement son Message concernant l'arrêté fédéral sur l'engagement en faveur de la paix de militaires de l'armée suisse dans la force multilatérale de l'Union européenne "European Union Force» (EUFOR) en Bosnie-Herzégovine ${ }^{1}$. Les Chambres fédérales ont chacune approuvé ce projet d'arrêté fédéral en octobre et en décembre 2004 et la Suisse a signé l'accord de son engagement dans l'EUFOR avec l'Union européenne le 22 décembre 2004.

La décision de remplacer l'actuelle force de stabilisation de l'OTAN en Bosnie (SFOR) par une force militaire européenne (EUFOR) avait été prise en décembre 2003 et confirmée lors du sommet de l'OTAN à Istanbul les 28 et 29 juin 2004. Les ministres des Affaires étrangères de l'Union européenne (UE) ont avalisé cette décision le 12 juillet 2004 à Bruxelles. L'EUFOR est opérationnelle depuis le 2 décembre 2004, après le feu vert accordé par le Conseil de sécurité de l'ONU ${ }^{2}$ pour le déploiement de cette force. Des contingents américains de l'OTAN restent tout de même sur place pour des opérations spéciales, en vertu d'un accord bilatéral avec l'UE.

* Par Xavier Tschumi Canosa, collaborateur scientifique à l'iuéd.

1 Conseil fédéral, Message concernant l'arrêté fédéral sur l'engagement en faveur de la paix de militaires de l'armée suisse dans la force multilatérale de l'Union européenne "European Union Force» (EUFOR) en Bosnie-Herzégovine du 26 mai 2004 (FF 2004 3063).

2 Conseil de sécurité de l'ONU, Résolution 1575 du 22 novembre 2004, doc. S/RES/1575 (2004). 
La mission de l'EUFOR est dénommée Althea et placée initialement sous commandement britannique. Elle est dotée d'un effectif d'environ 7000 hommes mais, si la situation le permet, le nombre de ses bataillons sera progressivement réduit de juin 2005 à fin 2006. L'effectif de l'EUFOR est composé d'équipes de liaison et d'observation (Liaison and Observation Teams - LOT) dotées chacune de huit militaires de même nationalité.

L'EUFOR reprendra les tâches militaires de la SFOR (garantir un environnement sûr en Bosnie-Herzégovine) mais aura aussi une mission civile de soutien aux autorités bosniaques, notamment en ce qui concerne le retour des réfugiés et la lutte contre le crime organisé. Elle sera donc associée plus étroitement que la SFOR aux activités du haut représentant de la communauté internationale sur place, Paddy Ashdown.

La Suisse participera à cette mission de l'EUFOR en mettant à la disposition de la brigade britannique au maximum deux LOT et quatre officiers supérieurs. Début novembre 2004, une première LOT et deux officiers d'état-major ont quitté la Suisse pour la Bosnie-Herzégovine. Le DDPS étudie encore la possibilité de mettre à la disposition de l'EUFOR des capacités de transport aérien.

\subsubsection{Promotion civile de la paix et renforcement des droits de l'homme}

Le 23 octobre 2002, le Conseil fédéral avait soumis au Parlement son Message concernant la loi fédérale sur des mesures de promotion civile de la paix et de renforcement des droits de l'homme.

Ded Annuaire 2003, $\mathrm{n}^{\circ}$ 1, sous-chap. 1.2 (pp.7-8).

Le projet de loi qui accompagnait ce message a été légèrement modifié puis adopté par les Chambres fédérales dans le courant de l'année 2003. La loi ellemême est entrée en vigueur le $1^{\text {er }}$ mai $2004^{3}$ et stipule en particulier que le financement des mesures de promotion civile de la paix et de renforcement des droits de l'homme est tiré de crédits-cadre pluriannuels. Le premier de ces créditscadre a été adopté par l'Assemblée fédérale en décembre 2003 et s'élève à 220 millions de francs pour une période d'au moins quatre ans à partir du $1^{\text {er }}$ janvier $2004^{4}$. L'aide financière octroyée par la Confédération au Centre Henry-Dunant pour le dialogue humanitaire, qui était régie par une loi fédérale propre, est désormais couverte par cette nouvelle loi $^{5}$.

\subsubsection{Commission extraparlementaire dans le domaine de la promotion de la paix}

Le 21 avril 2004, le Conseil fédéral a nommé les onze membres de la Commission extraparlementaire chargée des engagements militaires de la Suisse en faveur de la promotion internationale de la paix ${ }^{6}$. Cette commission est présidée

3 Assemblée fédérale, Loi fédérale sur des mesures de promotion civile de la paix et de renforcement des droits de l'homme du 19 décembre 2003, RS 193.9 (RO 2004 2157).

4 Assemblée fédérale, Arrêté fédéral concernant l'ouverture d'un crédit-cadre pour des mesures de gestion civile des conflits et de promotion des droits de l'homme du 17 décembre 2003 (FF 2004 2041).

5 «La loi fédérale du 15 décembre 2000 concernant la participation et l'octroi d'une aide financière de la Confédération au Centre Henry-Dunant pour le dialogue humanitaire est abrogée» (art. 11 de la Loi fédérale sur des mesures de promotion civile de la paix et de renforcement des droits de l'homme).

6 DDPS, communiqué de presse, 21 avril 2004. 
par l'ancien chef du Corps suisse d'aide en cas de catastrophe, Charles Raedersdorf, et doit conseiller le Département fédéral de la défense, de la protection de la population et des sports (DDPS) ainsi que le Département fédéral des affaires étrangères (DFAE) sur les questions politiques et conceptuelles soulevées par l'engagement de troupes suisses dans des opérations internationales de promotion de la paix. Les membres de cette commission sont nommés pour une durée de quatre ans, coïncidant avec la législature des Chambres fédérales.

\subsubsection{Projet de "Maison de la paix " à Genève}

Fin janvier 2004, le Conseil fédéral a confirmé vouloir poursuivre le projet «Maison de la paix » à Genève. A ses débuts, le projet en question se limitait à vouloir donner un toit à la formation de politique de sécurité qui allait devenir, en 1996, le «Centre de politique de sécurité, Genève»(GCSP). Avec la création subséquente de deux autres centres à Genève, le Centre international de déminage humanitaire (GICHD) et le Centre pour le contrôle démocratique des forces armées (DCAF), le projet s'est réorienté pour faire de la Maison de la paix un point de convergence de multiples acteurs, outre les trois centres précités, œuvrant dans les domaines de la paix et de la sécurité.

메 Annuaire 2003, n ${ }^{\circ}$ 1, sous-chap. 11.3 (pp. 201-202).

La Maison de la paix permettra d'intensifier les synergies entre ses occupants et, en apportant une dimension conceptuelle à la paix et la sécurité, pourra améliorer l'efficience des politiques internationales en la matière. Des économies financières sont également attendues de ce projet, en matière d'infrastructure et d'administration notamment. La Maison de la paix devrait être opérationnelle en 2008, en l'état actuel de la planification.

\subsection{Politique de sécurité}

\subsubsection{Politique de maîtrise des armements et de désarmement de la Suisse}

En réponse au postulat Haering ${ }^{7}$, le Conseil fédéral a approuvé début septembre 2004 le rapport sur la politique de maîtrise des armements et de désarmement (PMAD) de la Suisse ${ }^{8}$. Par les nombreuses actions qu'elle met en place, la PMAD suisse contribue à atteindre l'objectif de maintien et de promotion de la sécurité et de la paix que le Conseil fédéral s'est assigné dans son Rapport sur la politique extérieure 2000.

메 Annuaire 2001 (pp. 242-245).

Ce rapport présente tout d'abord le contexte international de sécurité dans lequel s'inscrit la PMAD suisse, puis les buts de cette dernière ainsi que les instruments dont elle dispose et, enfin, ses perspectives : la PMAD suisse vise à garantir la sécurité et la stabilité (nationales et internationales) au niveau

7 «Rapport sur le désarmement», Bulletin officiel, postulat 02.3541 déposé par Barbara Haering, 2 octobre 2002, invitant le Conseil fédéral «à présenter au Parlement, une fois par législature, un rapport sur les perspectives, les objectifs, les priorités, les instruments et les bases statistiques de sa politique de désarmement en relation avec les mesures visant à instaurer la confiance et la sécurité».

8 Conseil fédéral, Rapport du Conseil fédéral sur la politique de maîtrise des armements et de désarmement de la Suisse 2004 du 8 septembre 2004 (FF 2004 4833). 
d'armement le plus bas possible, en prévenant notamment la prolifération des armes de destruction massive ${ }^{9}$ et en travaillant à leur élimination totale. Un volet de cette politique concerne également le contrôle à l'exportation de telles armes et un autre les armes conventionnelles, dont les armes légères et de petit calibre ainsi que les mines, munitions et explosifs.

La PMAD que la Suisse met en œuvre «soutiendra en tout premier lieu les actions multilatérales de maîtrise des armements et de désarmement qui sont juridiquement contraignantes» et «soutiendra les solutions qui, dans la mesure du possible, associent tous les acteurs impliqués $»^{10}$.

\subsubsection{Partenariat pour la paix et Conseil de partenariat euro-atlantique de I'OTAN}

Le Partenariat pour la paix (PPP) a été créé en janvier 1994 par les 16 membres que comptait alors l'OTAN et fête en 2004 ses dix ans d'existence. Le PPP regroupe actuellement 30 pays, dont 10 sont entre-temps devenus membres de l'OTAN (3 en 1999 et 7 en 2004). La Suisse a rejoint le PPP le 11 décembre 1996.

Le PPP est le volet opérationnel du Conseil de partenariat euro-atlantique (CPEA) et en partage les objectifs: garantir la stabilité et minimiser les risques de conflits dans cette zone en améliorant la capacités des membres à agir de concert.

Le CPEA a été constitué en 1997 pour remplacer le Conseil de coopération nord-atlantique (CCNA) établi depuis 1991. Il compte 46 pays membres, dont les 26 membres de l'OTAN, et est le cénacle où se discutent les éléments de la politique de sécurité multilatérale et où les stratégies et lignes directrices sont décidées pour ensuite être mises en œuvre au travers du PPP. Les réunions du CPEA rassemblent tour à tour les ministres de la Défense et des Affaires étrangères, les chefs d'état-major et les ambassadeurs auprès de l'OTAN des pays membres de ce conseil. La Suisse est membre du CPEA depuis mai 1997.

La participation au PPP n'implique aucune obligation juridiquement contraignante pour les pays membres et ceux-ci décident bilatéralement avec l'OTAN des domaines de coopération dans lesquels ils veulent s'engager. Fin septembre 2003, la Suisse a remis à l'OTAN son programme de partenariat individuel (IPP) pour l'année $2004^{11}$, dans lequel elle expose sa politique de partenariat, les ressources qu'elle entend y consacrer (forces armées, moyens civils, moyens financiers, offres de formation) et les champs d'activités dans lesquels elle va œuvrer: gestion des crises, planification en cas d'urgence, pédagogie et instruction militaires, logistique et systèmes de communication et d'information notamment.

L'IPP de la Suisse pour l'année 2005 a été approuvé par le Conseil fédéral le 22 décembre 2004 et reprend largement les objectifs politiques et les moyens inscrits dans le programme précédent. La lutte contre le terrorisme international et la prolifération d'armes de destruction massive constituent de nouveaux champs thématiques de la collaboration de la Suisse au PPP.

$9 \quad$ Armes nucléaires, chimiques, biologiques et radiologiques ainsi que leurs vecteurs (missiles).

10 DFAE, Rapport du Conseil fédéral sur la politique de maîtrise des armements et de désarmement de la Suisse 2004, communiqué de presse, 8 septembre 2004.

11 Swiss Mission to NATO, Individual Partnership Programme between Switzerland and NATO for 2004, Brussels, 24 September 2003. Chaque IPP est normalement établi pour deux ans. 
Un des domaines importants dans lequel la Suisse s'est durablement et très tôt engagée au sein du PPP est celui des technologies de l'information: le forum du partenariat met par exemple en ligne toutes les informations sur les activités des membres du PPP et du CPEA (organisation pratique, documents de travail, archives, forums de discussion). Au travers du système PRIME ${ }^{12}$, certaines informations sont disponibles en temps réel (en particulier les IPP). Ce service est assuré par le Réseau sur les relations internationales et la sécurité ${ }^{13}$. Un autre exemple de l'engagement de la Suisse dans ce domaine est le développement par l'EPFZ de l'IMSMA ${ }^{14}$, un logiciel actuellement utilisé par quatre programmes d'action contre les mines sur cinq dans le monde. Le PPP constitue la plate-forme au sein de laquelle ce logiciel fait l'objet d'ateliers ${ }^{15}$.

En avril 2004, le Conseil fédéral a publié son rapport annuel 2003 sur la participation de la Suisse au CPEA et au $\mathrm{PPP}^{16}$. Cette participation s'est concrétisée par une vingtaine d'offres de cours et d'ateliers, une dizaine de manifestations et la livraison de matériel d'armée, principalement des véhicules. Les dépenses liées à cette participation se sont élevées en 2003 à près de 3,6 millions de francs, dont les neuf dixièmes à la charge du DDPS (DFAE pour le reste). Les dépenses de la SWISSCOY (contingent suisse au Kosovo) et des trois centres de Genève (voir ci-dessus le point 11.1.4) ne sont pas comprises dans ce montant car elles n'entrent que partiellement dans le cadre du PPP.

Le DFAE et le DDPS ont en outre conjointement publié deux brochures sur la Suisse et le Partenariat pour la paix, l'une en juillet et l'autre en septembre 2004'17.

\subsection{Mines antipersonnel et autres mines terrestres}

\subsubsection{Stratégie 2004-2007 de la Confédération en matière de mines antipersonnel}

Fin décembre 2003, le DFAE et le DDPS ont approuvé la stratégie 2004-2007 de la Confédération sur l'engagement de la Suisse en matière de mines antipersonnel ${ }^{18}$. Dans une première partie, ce document fait le point des lignes d'action déjà mises en place aux niveaux international (Convention d'Ottawa notamment) et national (coordination interdépartementale au sein de la Confédération, fondation du Centre international de déminage humanitaire, financement de programmes antimines, etc.). La stratégie est présentée dans la seconde partie du document.

12 Partnership Real-time Information Management and Exchange System.

13 International Relations and Security Network (ISN): ce réseau travaille sur mandat de la Confédération au sein du Center for Security Studies (CSS) de l'EPFZ.

14 Information Management System for Mine Action, logiciel développé pour le compte du Centre international de déminage humanitaire (GICHD).

15 Pour plus d'informations sur ces ateliers : <www.gichd.ch/imsma/training.htm>.

16 Conseil fédéral, Rapport annuel 2003 du Conseil fédéral sur la participation de la Suisse au Conseil de partenariat euro-atlantique et au Partenariat pour la paix, Berne, 21 avril 2004.

17 Centre de politique de sécurité internationale (CPSI - DFAE), Swiss Update. La politique étrangère de la Suisse et son engagement au sein du Partenariat pour la paix, Berne, DFAE; DDPS, juillet 2004, disponible sur demande à <zisp@eda.admin.ch>. Bureau interdépartemental CPEA/PPP, La Suisse et le Partenariat pour la paix, Berne, DFAE; DDPS, septembre 2004.

18 EDA/VBS, Strategie des Bundes 2004-2007 zum Personenminenengagement der Schweiz, Bern, 30. Dezember 2003. 
- Promouvoir Genève en tant que centre international de l'action antimines.

- Faire du GICHD un instrument prioritaire de la politique suisse en matière de mines et en diffuser sa reconnaissance internationale.

- Contribuer à élargir le cercle des pays parties à la Convention d'Ottawa et soutenir ces derniers dans la mise en ouvre de cette convention.

- Impliquer les acteurs armés non étatiques dans le respect des dispositions antimines.

- Renforcer l'engagement de la Suisse en matière d'action antimines au sein de l'ONU.

- Intégrer davantage la problématique des mines dans le concept de sécurité humaine.

\section{$\square$ Objectifs de la stratégie au niveau opérationnel}

- Maintenir et même augmenter le volume de l'aide financière destinée à la lutte antimines.

- Intégrer le plus possible les actions antimines dans les instruments de promotion de la paix déjà en œuvre.

- Promouvoir la mise en place et le développement des structures et capacités de lutte contre les mines dans les pays mêmes qui sont affectés.

- Veiller à assurer la cohérence de la politique suisse en matière de mines.

Au niveau de l'organisation des tâches au sein de la Confédération, la stratégie prévoit de répartir les domaines de l'action antimines entre la direction politique du DFAE (pour le développement et la coordination de la politique suisse en matière de mines et pour les projets de déminage humanitaire), la DDC (pour l'aide aux victimes de mines et la prévention contre les mines dans le cadre de ses propres projets et programmes de coopération ou d'aide humanitaire) et la direction de la politique de sécurité du DDPS (pour la mise à disposition de personnel et de matériel et pour le soutien financier au GICHD).

Selon la stratégie, les moyens financiers disponibles pour les activités antimines de la Suisse seront légèrement augmentés dans les années à venir, passant d'environ 15 à 16 millions de francs par an. En 2004, les trois quarts de ces moyens ont financé des projets de déminage, le quart restant ayant financé des activités d'aide aux victimes de mines, de prévention et de politique antimines.

La mise en œuvre de la stratégie passe, sur le plan des objectifs politiques, par un soutien financier continu au GICHD (environ 8 millions de francs par an), l'organisation alternée entre Genève et un pays touché par les mines des conférences annuelles des Etats parties à la convention d'Ottawa, la fondation d'un groupe de réflexion sur les acteurs non étatiques dans la problématique des mines, le soutien politique et financier à l'Appel de Genève ${ }^{19}$, des initiatives visant à universaliser les prescriptions de la convention d'Ottawa et l'institutionnalisation des tables rondes bisannuelles entre la Confédération et les ONG suisses actives dans la lutte contre les mines. Sur le plan des objectifs opérationnels, la stratégie se mettra en place par un financement durable et régulier des projets de déminage, par des projets de prévention contre les mines et d'assistance aux victimes ainsi que par la destruction des stocks de mines.

19 L'Appel de Genève est une organisation dont le but est d'impliquer les acteurs non étatiques dans le respect des normes humanitaires, en particulier celle sur l'interdiction des mines antipersonnel. 


\subsubsection{Conférence d'examen de la convention d'Ottawa sur l'interdiction des mines antipersonnel}

La convention d'Ottawa ${ }^{20}$ sur l'interdiction des mines antipersonnel a été ouverte à la signature des pays dès le 3 décembre 1997 et est entrée en vigueur le $1^{\text {er }}$ mars 1999. La Suisse l'a signée le premier jour et l'a ratifiée le 24 mars 1998. Elle a terminé la destruction de ses stocks de mines au début de l'année suivante (elle avait renoncé à l'utilisation des mines depuis 1995 déjà).

Le baromètre des ratifications, proposé par l'organisation Handicap International, indique que depuis le 3 décembre 1997, 144 Etats ont signé et ratifié le traité (ou alors y ont adhéré), mais que seulement 51 Etats ont procédé à la destruction totale de leurs stocks (chiffres de décembre 2004) ${ }^{21}$.

Cinq ans après l'entrée en vigueur de la Convention, le secrétaire général des Nations unies a convoqué à Nairobi la première conférence d'examen de cette convention, du 29 novembre au 3 décembre 2004 (Sommet de Nairobi pour un monde sans mines ${ }^{22}$ ). La préparation du sommet de Nairobi a débuté en juin 2002 déjà et s'est formalisée par deux réunions préparatoires qui se sont déroulées en février et en juin 2004 dans les bâtiments de l'ONU à Genève. Les deux objectifs de cette conférence étaient de faire le point des progrès réalisés depuis l'entrée en vigueur du traité et d'établir un plan d'action concret pour les cinq années à venir et au-delà. Si les progrès sont indéniables sur tous les plans de la convention (partie II du rapport final de la conférence ${ }^{23}$ ), il en reste encore beaucoup à réaliser: mettre fin aux souffrances causées par les mines antipersonnel est le titre du plan d'action de Nairobi pour les années 2005 à 2009 (partie III du rapport final de la conférence). Ce plan d'action en 70 points vise à universaliser l'adhésion à la convention d'Ottawa, à détruire les stocks de mines antipersonnel, à déminer les zones affectées par ces engins et à assister les victimes. La coopération, l'assistance, la transparence et l'échange d'information entre les Etats parties entrent aussi dans le plan d'action, de même que les mesures visant à faciliter le respect des termes de la convention et le soutien à sa mise en œuvre. La déclaration de Nairobi pour un monde sans mines qui accompagne ce plan d'action en constitue une sorte de résumé politique sur deux pages (partie IV du rapport final de la conférence).

La délégation suisse à Nairobi était conduite par le secrétaire d'Etat aux Affaires étrangères, Franz von Däniken, ce qui a été critiqué notamment par la Campagne suisse contre les mines antipersonnel, qui regrette que Micheline CalmyRey, la cheffe du DFAE, n'ait pas fait elle-même le déplacement, alors que Genève apparaît de plus en plus comme la capitale mondiale de la lutte contre les mines ${ }^{24}$. Sur les 135 Etats ayant participé à la conférence, seuls 31 ont été représentés au niveau ministériel, dont le Canada et la France.

20 Convention sur l'interdiction de l'emploi, du stockage, de la production et du transfert des mines antipersonnel et sur leur destruction (Traité d'Ottawa).

21 Ce baromètre est actualisé constamment sur le site de Handicap International: <www.handicapinternational.org >, taper «baromètre» dans le champ «Rechercher».

22 Le site Internet officiel du sommet de Nairobi est <www.reviewconference.org $>$.

23 Le document est disponible sur le site du sommet (référence APLC/CONF/2004/5).

24 «La Suisse, autrefois à la pointe du combat, fait profil bas à Nairobi », Le Temps, 27 novembre 2004. 


\subsubsection{Groupe de soutien à l'action contre les mines de I'ONU}

La Suisse, en la personne du chef de la mission permanente de la Suisse à l'ONU, préside depuis janvier 2004 et pour deux ans le Groupe de soutien à l'action contre les mines de l'ONU (MASG). Ce groupe informel, qui rassemble 26 pays membres, est un des principaux instruments de mobilisation des ressources de l'action antimines de l'ONU. Pour ses membres, c'est un forum d'échange d'informations et de coordination, et, pour les pays affectés par les mines, une tribune à laquelle ils peuvent présenter leur situation et faire le point sur les programmes de déminage en cours ou à mettre en œuvre. Les informations recueillies au sein de ce groupe circulent également jusqu'au Secrétariat de l'ONU. Depuis qu'elle préside le MASG, la Suisse a pris l'initiative de publier mensuellement une newsletter ${ }^{25}$ qui présente de manière inofficielle le contenu des réunions du groupe et l'avancement des programmes antimines menés principalement par l'UNMAS (pour les situations d'urgence et les opérations de maintien de la paix), l'UNICEF (pour les activités de sensibilisation au problème des mines) et le PNUD (pour les programmes à plus long terme) dans différents pays.

\subsubsection{Le CICR et les mines terrestres}

Le Comité international de la Croix-Rouge (CICR) s'est très tôt engagé dans la lutte contre les mines antipersonnel, son appel pour leur interdiction datant de février 1994, soit huit mois après celui de la Campagne internationale pour l'interdiction des mines terrestres (ICBL). L'effort commun de ces deux organisations, de l'ONU et de nombreux États a conduit à l'adoption de la convention d'Ottawa (voir ci-dessus) en 1997.

En octobre 1999, la même année qu'est entrée en vigueur cette convention, le Mouvement international de la Croix-Rouge et du Croissant-Rouge a adopté sa stratégie concernant les mines terrestres. Cette stratégie sur cinq ans s'articule autour de deux lignes d'action complémentaires: premièrement, assister et protéger les populations civiles affectées par ces mines et, deuxièmement, promouvoir l'interdiction des mines terrestres (pour parvenir, à terme, à leur élimination).

Par la suite, la préoccupation du Mouvement concernant les conséquences humanitaires des mines antipersonnel s'est élargie aux débris de guerre explosifs $^{26}$. En 2001, cette préoccupation s'est muée en résolution qui invitait les Etats parties à la Convention de 1980 sur certaines armes classiques (CCAC) à négocier les termes d'un instrument additionnel à la convention concernant ces débris. La négociation a formellement débuté lors de la $2^{\mathrm{e}}$ Conférence d'examen de la CCAC en décembre 2001 et a trouvé un épilogue à Genève le 28 novembre 2003 sous la forme du protocole V relatif aux débris de guerre explosifs. En outre, lors de la $2^{\mathrm{e}}$ Conférence d'examen de la CCAC, la portée de la convention a été étendue aux conflits armés de caractère non international (amendement de l'article premier de la convention), décision également contenue dans la résolution 8 du Conseil des délégués du Mouvement en novembre

25 Ces newsletters sont disponibles sur le site Internet de la Mission permanente de la Suisse auprès des Nations unies : <www.eda.admin.ch/newyork_miss/e/home/masg.html>.

26 Munitions explosives (non explosées ou abandonnées) qui subsistent dans une zone après la fin d'un conflit. 
$2001^{27}$. La Suisse a ratifié cet amendement le 19 janvier 2004 (sur la base de l'arrêté fédéral du 15 décembre $2003^{28}$ ). Le protocole $\mathrm{V}$ doit encore être soumis au Parlement suisse pour approbation.

Le Conseil des délégués du Mouvement a décidé29, fin 2003, de prolonger la période de mise en ouvre de sa stratégie jusqu'en 2009 et d'en étendre la portée à l'ensemble des débris de guerre explosifs. En outre, le CICR présentera au prochain Conseil des délégués en 2005 un rapport sur les progrès de la stratégie.

\subsection{Armes de destruction massive (ADM)}

\subsubsection{Résolution du Conseil de sécurité de I'ONU sur la non-prolifération des ADM}

Un petit groupe de pays ${ }^{30}$, dont la Suisse, a été à l'origine d'un débat public du Conseil de sécurité de l'ONU sur les armes de destruction massive (ADM), le 22 avril 2004. Un projet de résolution sur la non-prolifération de ces armes parmi les acteurs non étatiques était déjà en négociation entre les seuls membres permanents du Conseil de sécurité depuis septembre 2003. Cependant, au vu des implications contraignantes de ce projet pour tous les Etats membres de l'ONU, ce petit groupe de pays a réclamé davantage de transparence, de clarté et d'équilibre dans le texte. Il regrettait par exemple que le projet de résolution ne contienne qu'une référence incidente au désarmement, alors que les armes biologiques et chimiques sont interdites par des traités internationaux et que les pays qui possèdent des armes nucléaires ont manifesté sans équivoque leur volonté d'éliminer leur arsenal. Un autre souci de ce petit groupe de pays était l'ancrage de ce projet de résolution dans le chapitre VII ${ }^{31}$ de la Charte des Nations unies: la Suisse et le Brésil, en particulier, se sont exprimés pour que la résolution ne puisse être comprise comme une autorisation de recours unilatéral à la force en cas de non-respect des obligations inscrites dans le texte.

Une semaine après ce débat public au sein du Conseil de sécurité de l'ONU, ce dernier a unanimement adopté la résolution $1540^{32}$, par laquelle tous les Etats doivent prendre toutes les dispositions nécessaires afin de prévenir que des acteurs non étatiques ne mettent au point, ne fabriquent, ne se procurent ou utilisent des ADM ainsi que leurs vecteurs. Le texte de cette résolution prend partiellement en compte les demandes du petit groupe de pays à l'origine du débat

27 Conseil des délégués 2001 du Mouvement international de la Croix-Rouge et du Croissant-Rouge, 11-14 novembre 2001, Résolution 8-Les débris de guerre explosifs et les conflits armés de caractère non international.

28 Assemblée fédérale, Arrêté fédéral concernant l'amendement du 21 décembre 2001 de l'art. 1 de la Convention sur l'interdiction ou la limitation de l'emploi de certaines armes classiques qui peuvent être considérées comme produisant des effets traumatiques excessifs ou comme frappant sans discrimination du 15 décembre 2003 (RO 2004 3951).

29 Conseil des délégués 2003 du Mouvement international de la Croix-Rouge et du Croissant-Rouge, 30 novembre-2 décembre 2003, Résolution 11 - Les débris de guerre explosifs et la stratégie du Mouvement concernant les mines.

30 Afrique du Sud, Canada, Mexique, Nouvelle-Zélande, Suède, Suisse.

31 Ce chapitre règle 1' «action en cas de menace contre la paix, de rupture de la paix et d'acte d'agression ».

32 Conseil de sécurité de l'ONU, Résolution 1540 du 28 avril 2004, doc. S/RES/1540 (2004). 
public, notamment en ce qui concerne son interprétation par rapport aux textes des traités et conventions sur les ADM.

\subsubsection{Partenariat mondial contre la prolifération des ADM}

Le Partenariat mondial contre la prolifération des armes de destruction massive, fondé à l'initiative des Etats du G-8 en juin 2002 et auquel la Suisse s'est jointe en mai 2003, vise principalement à éviter que tout ou partie de la technologie des $\mathrm{ADM}$ ne tombe entre les mains des acteurs non étatiques mentionnés ci-dessus.

And Annuaire 2004, $\mathrm{n}^{\circ}$ 1, sous-chap. 11.4. (p. 206).

Le rapport annuel 2004 de ce partenariat $^{33}$ indique que la Suisse ainsi que cinq autres pays ${ }^{34}$ se sont engagés à hauteur de 200 millions de dollars pour des projets entrant dans le cadre des principes du partenariat mondial, essentiellement pour le désarmement chimique de la Fédération de Russie (voir ci-dessous). L'engagement financier de la Suisse est puisé dans le crédit-cadre de 17 millions de francs prévu par l'arrêté fédéral du 3 mars $2003^{35}$.

\subsubsection{Désarmement chimique}

La Suisse et la Fédération de Russie ont signé à Moscou, le 28 janvier 2004, une convention bilatérale sur le soutien de la Suisse à la destruction des stocks russes d'armes chimiques. Cet accord règle les conditions générales des projets de désarmement chimique financés par la Suisse dans ce pays, pour lesquels 15 millions de francs seront consacrés dans les cinq années à venir.

Sans ces projets (et ceux que de nombreux autres pays mettent aussi en œuvre), l'élimination de l'imposant arsenal chimique russe (40'000 tonnes) ne pourrait être assurée avant 2012, délai prescrit par la Convention sur les armes chimiques (CAC) de 1993. Les États-Unis, qui disposent d'un stock de 31'500 tonnes, en ont déjà détruit 8600 tonnes, alors que seulement 640 tonnes des stocks russes ont été éliminées, grâce notamment aux projets mis en œuvre dans le cadre du Partenariat mondial contre la prolifération des armes de destruction massive (voir ci-dessus).

L'organisation Green Cross Suisse a été mandatée une nouvelle fois en 2004 par le Centre de politique et de sécurité internationale (CPSI) et la DDC (tous deux rattachés au DFAE) pour poursuivre les projets suisses de désarmement chimique dans la Fédération de Russie. La Confédération soutient financièrement Green Cross Suisse depuis 1994.

33 Rapport annuel du Partenariat mondial du G-8. Groupe de haut niveau du $G-8$, Sommet des chefs d'Etat et de gouvernement du G-8, Sea Island, 10 juin 2004, disponible sur <www.g8.utoronto.ca/ francais>.

34 Finlande, Norvège, Pays-Bas, Pologne et Suède. Comme la Suisse, ces cinq pays se sont joints au partenariat en 2003.

35 Assemblée fédérale, Arrêté fédéral relatif au soutien au désarmement chimique universel du 3 mars 2003 (FF 2003 4664). 


\section{SOURCES}

CICR, Mettre fin à l'ère des mines. Succès et défis, Genève, juin 2004, <www.icrc.org/Web/fre/sitefre0. nsf/htmlall/p0846/\$File/ICRC_001_0846.PDF?Open>.

Conseil fédéral, Rapport du Conseil fédéral sur la politique de maîtrise des armements et de désarmement de la Suisse 2004 du 8 septembre 2004 (FF 2004 4833).

DDPS, Départ d'une équipe suisse d'observation pour la Bosnie-Herzégovine dans le cadre de l'EUFOR, communiqué de presse, 2 novembre 2004.

Le Temps, «Armes de destruction massive: offensive suisse à 1'ONU », 22 avril 2004.

\section{SITES INTERNET}

Appel de Genève: <www.genevacall.org>.

Centre de politique de sécurité, Genève (GCSP): <www.gcsp.ch $>$.

Centre international de déminage humanitaire - Genève (GICHD) : <www.gichd.ch $>$.

Centre pour un contrôle démocratique des forces armées (DCAF) : $<$ www.dcaf.ch $>$.

Comité international de la Croix-Rouge: $<w w w . i c r c . o r g>$.

Green Cross Suisse: <www.greencross.ch>.

Service des NU pour l'action antimines (UNMAS) : <www.mineaction.org $>$.

La Suisse et le Partenariat pour la paix (site officiel tenu par le DFAE et le DDPS) : <www.pfp.admin.ch $>$.

Mission suisse auprès de l'OTAN (Partenariat pour la paix) : <www.nato.int/pfp/ch $>$.

International Relations and Security Network (ISN) : $<$ www.isn.ch $>$.

Center for Security Studies (CSS) : <www.css.ethz.ch $>$. 